TRANSACTIONS OF THE

AMERICAN MATHEMATICAL SOCIETY

Volume 356, Number 11, Pages 4623-4642

S 0002-9947(04)03504-4

Article electronically published on April 27, 2004

\title{
REAL LOCI OF SYMPLECTIC REDUCTIONS
}

\author{
R. F. GOLDIN AND T. S. HOLM
}

\begin{abstract}
Let $M$ be a compact, connected symplectic manifold with a Hamiltonian action of a compact $n$-dimensional torus $T$. Suppose that $M$ is equipped with an anti-symplectic involution $\sigma$ compatible with the $T$-action. The real locus of $M$ is the fixed point set $M^{\sigma}$ of $\sigma$. Duistermaat introduced real loci, and extended several theorems of symplectic geometry to real loci. In this paper, we extend another classical result of symplectic geometry to real loci: the Kirwan surjectivity theorem. In addition, we compute the kernel of the real Kirwan map. These results are direct consequences of techniques introduced by Tolman and Weitsman. In some examples, these results allow us to show that a symplectic reduction $M / / T$ has the same ordinary cohomology as its real locus $(M / / T)^{\sigma_{\text {red }}}$, with degrees halved. This extends Duistermaat's original result on real loci to a case in which there is not a natural Hamiltonian torus action.
\end{abstract}

\section{INTRODUCTION}

Let $M$ be a compact symplectic manifold, and suppose an $n$-dimensional torus $T$ acts on $M$ in a Hamiltonian fashion. Let $\Phi: M \rightarrow \mathfrak{t}^{*}$ be a moment map. The components of the moment map are (equivariant) Morse-Bott functions on $M$, and hence may be used to determine the (equivariant) topology of $M$.

Now suppose that $\sigma$ is an anti-symplectic involution on $M$ that anti-commutes with the $T$ action:

$$
\sigma(t \cdot x)=t^{-1} \cdot \sigma(x)
$$

for all $t \in T$ and $x \in M$. We assume that $M^{\sigma}$ is nonempty. We call the fixed point set $Q=M^{\sigma}$ of $\sigma$ the real locus of $M$. The real locus is a Lagrangian submanifold of $M$. The motivating example of a real locus is a complex variety $M$ under complex conjugation $\sigma$. In this case, the real locus is the set of real points on the variety. By (1.1) the subgroup of $T$ of elements of order 2 acts on $Q$. We call this subgroup $T_{\mathbb{R}}$, the real torus in $T$. It is immediate that $T_{\mathbb{R}} \cong(\mathbb{Z} / 2 \mathbb{Z})^{n}$.

Duistermaat [11] proved that the real locus has full moment image, that is, that $\Phi(M)=\Phi(Q)$. Moreover, he showed that the components of the moment map are Morse-Bott functions for the real locus, when using $\mathbb{Z} / 2 \mathbb{Z}$ coefficients, and so we can understand the topology of $Q$ via the moment map. Biss, Guillemin

Received by the editors April 4, 2003 and, in revised form, July 24, 2003.

2000 Mathematics Subject Classification. Primary 53D20.

Key words and phrases. Real locus, symplectic reduction.

The first author was partially supported by NSF grant DMS-0305128. This research was partially conducted during the period when the second author served as a Clay Mathematics Institute Liftoff Fellow. The second author was also partially supported by an NSF postdoctoral fellowship. 
and the second author [6] proved that these moment map components can also be used to understand the equivariant topology of the real locus with respect to the restricted $T_{\mathbb{R}}$ action. In addition, Sjamaar and O'Shea have generalized the results of Duistermaat for Hamiltonian actions of nonabelian Lie groups [16]. The principle behind these results is that real loci should behave in a fashion similar to the symplectic manifolds of which they are submanifolds. We show that this philosophy applies in the context of symplectic reductions.

Given a compact Hamiltonian $T$-space $M$, Kirwan 14 proved that when $T$ acts freely on $\Phi^{-1}(\mu)$, the inclusion map $\Phi^{-1}(\mu) \hookrightarrow M$ induces a surjection in equivariant cohomology with rational coefficients:

$$
\kappa: H_{T}^{*}(M) \rightarrow H_{T}^{*}\left(\Phi^{-1}(\mu)\right)=H^{*}(M / / T(\mu)) .
$$

The map $\kappa$ is called the Kirwan map. In [19] the authors note that under reasonable assumptions about the torsion of the fixed point sets and the group action, this map is surjective over the integers as well. For $T=S^{1}$ one can state the result as follows. For any prime $p$, assume that either there is no $p$-torsion in the $\mathbb{Z}$-cohomology of the fixed point set $M^{T}$, or every point not in $M^{T}$ has a free $\mathbb{Z} / p \mathbb{Z}$ action. Then the map (1.2) is surjective with integer coefficients. For higher dimensional $T$, the authors assume the action is quasi-free to prove Kirwan's surjectivity over $\mathbb{Z}$, although this is stronger than necessary. One natural question is, what is the kernel of $\kappa$ ? This question was answered in [19] and refined by the first author in the case of rational coefficients [12].

Suppose that $M$ has the additional structure of an anti-symplectic involution, compatible with torus action as specified above. Suppose also that $Q:=M^{\sigma}$ is nonempty. In this article we prove a surjectivity result for real loci analogous to Kirwan's result (Theorem 1). We also use Tolman and Weitsman's equivariant Morse theoretic methods to compute the kernel of this real version of the Kirwan map (Theorem 2). Thus the main contribution of this article is to complete the program begun by Duistermaat in showing that the real locus of a Hamiltonian $T$-space has a $(\mathbb{Z} / 2 \mathbb{Z})^{n}$-action which behaves as if it were itself a Hamiltonian $T$ space. Not only is the equivariant cohomology ring of $Q$ described by restrictions to fixed points, but there is a well-defined notion of "real reduction" and the major theorems about the cohomology rings of reduced spaces go through in the real case. The proofs we present are straightforward extensions of the work of Atiyah-Bott, Kirwan, and Tolman-Weitsman to the real case. We then explore the ramifications of real reduction and these theorems in a series of examples.

The key to Kirwan's proof of surjectivity is the analysis of the function

$$
\|\Phi\|^{2}: M \rightarrow \mathbb{R} \text {. }
$$

In the case of $S^{1}$, this is not a Morse function, but it is Morse-Bott except at 0 , the function's minimum value. For $\operatorname{dim} T>1$, there are a finite number of critical values of $\Phi$ where $\|\Phi\|^{2}$ is not Morse-Bott, in addition to the minimum. Moreover,

$$
\left(\|\Phi\|^{2}\right)^{-1}(0)=\Phi^{-1}(0) .
$$

Kirwan uses the critical sets of $\|\Phi\|^{2}$ to argue inductively (on the critical sets) that the equivariant cohomology of $M$ surjects onto the ordinary cohomology of $M / / T(0)$. It is clear that surjectivity for general values $\mu$ of $\Phi$ follows by shifting the moment map by an appropriate constant. These arguments easily go through when the function is restricted to the real locus, as we will see. 
A fundamental step in the proof of surjectivity and the computation of the kernel is a lemma due to Atiyah and Bott [3]. This lemma describes the local topology, in and around a fixed point component of $M^{T}$. Under the hypothesis of no 2-torsion (Definition 1.1) we show that this lemma still holds for $(\mathbb{Z} / 2 \mathbb{Z})^{n}$-cohomology with $\mathbb{Z} / 2 \mathbb{Z}$ coefficients. We then apply the lemma and an inductive argument to both the question of surjectivity and to the computation of the kernel for real loci.

For the rest of this article, let $M$ be a compact, connected symplectic manifold, endowed with a Hamiltonian action of a compact torus $T$ and moment map $\Phi$. Let $\sigma$ be an anti-symplectic involution, anti-commuting with the torus action, and let $T_{\mathbb{R}} \subset T$ be the set of order 2 elements, plus the identity. Recall that $T_{\mathbb{R}}$ acts on $Q$. We begin with a definition.

Definition 1.1. Let $p \in M$ be a critical point of $\Phi$. Let $H \subset T$ be the maximal connected subtorus of $T$ fixing $p, \Lambda_{T}$ the weight lattice of $T$, and $\Lambda_{H}$ the weight lattice of $H$. Let $N$ be the connected component of $M^{H}$ containing $p$. We denote by $\alpha_{1}, \ldots, \alpha_{k}$ the weights of the $H$ action on $\nu_{p} N$, the fiber over $p$ of the normal bundle to $N$ in $M$, where $k=\operatorname{dim} \nu_{p} N$. We say that $p$ is a 2-torsion point if $\alpha_{i} \equiv 0$ $\bmod 2 \Lambda_{H}$ for some $i=1, \ldots, k$.

Remark 1.2. Suppose $M$ has a symplectic involution $\sigma$ with real locus $Q$. Suppose $p$ is critical for $\Phi$, fixed by $H$, and $N$ is the connected component of $M^{H}$ containing $p$. If $p$ lies in $Q$, the $H$ action on the normal bundle to $N$ in $M$ restricts to an $H_{\mathbb{R}}$ action on the normal bundle $\nu_{Q} N^{\sigma}$ to $N^{\sigma}$ in $Q$. Let $\beta_{1}, \ldots, \beta_{k}$ be the weights of the irreducible representations obtained by this action on the fiber over $p$. Then $p$ is a 2 -torsion point if and only if $\beta_{i}$ is trivial for some $i$.

Remark 1.3. The functions $\|\Phi\|^{2}$ and a family of perturbations (see Section (5) have critical sets fixed by various subtori of $T$. Tolman and Weitsman prove $H_{T}^{*}(M ; \mathbb{Z}) \rightarrow$ $H^{*}(M / / T ; \mathbb{Z})$ is a surjection provided that $T$ acts quasi-freely. As they note, one needs to ensure that the negative normal bundles to all critical sets of these functions have nontrivial equivariant Euler classes. For the real locus case, "no 2-torsion points" plays the same role as "quasi-free" does in the symplectic case. At the critical sets of this family of functions, the negative normal bundles have top StiefelWhitney classes which are nontrivial in the $\left(H_{T_{\mathbb{R}}}^{*} \otimes 1\right)$-component of $H_{T_{\mathbb{R}}}^{*}(N)$ for each connected component $N$ of the critical set. This amounts to the requirement that, over any point in $N$, the negative normal bundle splits into one-dimensional nontrivial representations of $T_{\mathbb{R}}$. The condition that $Q$ contain no 2-torsion points ensures this for the family of perturbations of $\|\Phi\|^{2}$.

The first main theorem is the surjectivity analogue of (1.2) for real loci.

Theorem 1. Suppose $M$ is a compact symplectic manifold with a Hamiltonian $T^{n}$-action, and that $Q$ is the real locus of $M$. Let $\Phi$ be a moment map on $M$ and $\mu$ a regular value of $\Phi$. Suppose further that $T^{n}$ acts freely on $\Phi^{-1}(\mu)$ and that $Q$ contains no 2-torsion points. Then the real Kirwan map in $T_{\mathbb{R}}$-equivariant cohomology with $\mathbb{Z} / 2 \mathbb{Z}$ coefficients

$$
\kappa_{\mathbb{R}}: H_{T_{\mathbb{R}}}^{*}(Q) \rightarrow H_{T_{\mathbb{R}}}^{*}\left(\left.\Phi\right|_{Q} ^{-1}(\mu)\right)=H^{*}\left(Q / / T_{\mathbb{R}}(\mu)\right),
$$

induced by inclusion, is a surjection.

We omit the superscript $n$ in $T^{n}$ when the dimension is clear. 
Remark 1.4. The hypothesis that the real locus have no 2-torsion points is reasonably strong. Real loci of toric varieties and coadjoint orbits in type $A_{n}$ satisfy this hypothesis, for example, but the real loci of maximal coadjoint orbits in type $B_{n}$ do not.

The next task we complete is to specify the kernel of $\kappa_{\mathbb{R}}$. The kernel is precisely the real analog of the kernel found in [19]. For every $\xi \in \mathfrak{t}$, let

$$
Q_{\xi}=\{p \in Q \mid\langle\Phi(p), \xi\rangle \leq 0\} .
$$

Let $F=M^{T}$ denote the fixed point set, and let

$$
K_{\xi}=\left\{\alpha \in H_{T_{\mathbb{R}}}^{*}(Q ; \mathbb{Z} / 2 \mathbb{Z})|\alpha|_{F \cap Q_{\xi}}=0\right\} .
$$

Finally, define the ideal

$$
K_{\mathbb{R}}=\left\langle\sum_{\xi \in \mathfrak{t}} K_{\xi}\right\rangle .
$$

Theorem 2. Suppose $M$ is a compact symplectic manifold with a Hamiltonian Taction, an anti-symplectic involution $\sigma$ anti-commuting with $T$, and real locus $Q$. Let $\Phi$ be a moment map on $M$ and $\mu$ a regular value of $\Phi$ such that $T$ acts freely on $\Phi^{-1}(\mu)$ and that $Q$ contains no 2-torsion points. Then the kernel of the real Kirwan map is the ideal $K_{\mathbb{R}}$, so there is a short exact sequence

$$
0 \rightarrow K_{\mathbb{R}} \rightarrow H_{T_{\mathbb{R}}}^{*}(Q) \rightarrow H^{*}\left(Q / / T_{\mathbb{R}}(\mu)\right) \rightarrow 0,
$$

where the cohomology is taken with $\mathbb{Z} / 2 \mathbb{Z}$ coefficients.

The remainder of this paper is organized as follows. In Section 2, we review $G$-equivariant cohomology with specific attention to the case of $\mathbb{Z} / 2 \mathbb{Z}$ coefficients and $G=(\mathbb{Z} / 2 \mathbb{Z})^{n}=T_{\mathbb{R}}$. Here we make explicit the Thom isomorphism theorem and the $\mathbb{Z} / 2 \mathbb{Z}$ version of the Atiyah-Bott lemma. In Section 3 , we study MorseKirwan theory on real loci. In Section 4 we discuss reduction, an induced antisymplectic involution on the symplectic reduction, and we prove the surjectivity theorem (Theorem 1). In Section [5 we prove Theorem 2 the description of the kernel of the real Kirwan map. Finally, in Section 6 6 we work out several pertinent examples. In particular, we present an example of a symplectic reduction which has the same cohomology as its real locus, with degrees divided in half. As $M / / T$ does not in general have a torus action, this example generalizes Duistermaat's original work on real loci, in which he uses the $T$ action to make an analogous statement for $M$ and its real locus.

The authors would like to thank Victor Guillemin, Robert Kleinberg, Dan Dugger and Reyer Sjamaar for useful comments during the preparation of this paper.

\section{Notes ON EQUiVARIANT $\mathbb{Z} / 2 \mathbb{Z}$-COHOMOLOGY}

In this section we discuss equivariant cohomology and the Thom isomorphism in $\mathbb{Z} / 2 \mathbb{Z}$ coefficients. We then state a topological lemma (Lemma 2.1) that plays an important role in the proofs of Theorems 1 and 2. Given a bundle $E \rightarrow B$ with a $\mathbb{Z} / 2 \mathbb{Z}$ action exactly fixing $B$, we show the top Stiefel-Whitney class $w_{k}(E)$ is not a zero-divisor in $H_{\mathbb{Z} / 2 \mathbb{Z}}^{*}(B ; \mathbb{Z} / 2 \mathbb{Z})$. Similarly, given a $T_{\mathbb{R}}$ action on a space $E$ that has no 2 -torsion, we show that $w_{k}(E) \in H_{T_{\mathbb{R}}}(B ; \mathbb{Z} / 2 \mathbb{Z})$ is not a 0 -divisor. This result implies that a certain long exact sequence splits into short exact sequences 
(Lemma 2.3) allowing inductive arguments to be applied. This is not surprising given the analogous result due to Tolman and Weitsman [19] for the $T$-equivariant cohomology of $B$, where $T$ is a compact torus acting on $E$ and fixing $B$, and the cohomology is taken with integer coefficients.

2.1. Equivariant cohomology with $\mathbb{Z} / 2 \mathbb{Z}$ coefficients. Let $G$ be a compact Lie group acting on $M$. Then the equivariant cohomology of $M$ is defined to be the ordinary cohomology of the Borel construction. Let $E G$ be an equivariantly contractible space on which $G$ acts freely. The Borel construction is

$$
M_{G}:=M \times_{G} E G
$$

where the right-hand side is the set of equivalence classes of pairs $(m, e)$ such that $m \in M, e \in E G$ and $(m, e) \sim(g \cdot m, g \cdot e)$ for all $g \in G$. By definition, the $G$-equivariant cohomology of $M$ is $H_{G}^{*}(M):=H^{*}\left(M_{G}\right)$. All $M_{G}$ are homotopic for different choices of $E G$, and so any choice of $E G$ will induce the same equivariant cohomology ring.

For any compact oriented $G$-manifolds $N$ and $M$ and any $G$-equivariant map $f: N \rightarrow M$, there is an associated map $f_{G}: N_{G} \rightarrow M_{G}$ defined by $f_{G}(n, e)=$ $(f(n), e)$ on equivalence classes. This induces the pullback and the pushforward in cohomology:

$$
\begin{aligned}
& f^{*}: H_{G}^{*}(M) \rightarrow H_{G}^{*}(N) \\
& f_{*}: H_{G}^{*}(N) \rightarrow H_{G}^{*-q}(M)
\end{aligned}
$$

where $q=\operatorname{dim} N-\operatorname{dim} M$, and may be negative. In particular, equivariant cohomology is functorial. Note that the pushforward does not preserve degree, whereas the pullback is a ring map.

In the case of the map $\pi: M \rightarrow p t$ which sends all of $M$ to a point $p t$, the induced map in equivariant cohomology $\pi^{*}: H_{G}^{*}(p)=H^{*}(E G / G) \rightarrow H_{G}^{*}(M)$ turns $H_{G}^{*}(M)$ into a module over $H_{G}^{*}:=H_{G}^{*}(p t)$. We note that $H_{G}^{*}(M)$ is not always a free module over $H_{G}^{*}$ : in particular, if $G$ acts freely on a finite dimensional manifold $M$, then $H_{G}^{*}(M)=H^{*}(M / G)$ (as can be seen by noticing that the fiber of $M_{G} \rightarrow M / G$ is $E G$, which is contractible) has finite-dimensional cohomology. However, $H_{G}^{*}$ is not finite for nontrivial $G$, and so the module is not free. When $H_{G}^{*}(M)=$ $H_{G}^{*} \otimes H^{*}(M)$ as vector spaces, we say that $M$ is equivariantly formal. Clearly, the module structure is free in this case. Hamiltonian $G$-spaces are examples of equivariantly formal spaces.

An important fact is that for $G=(\mathbb{Z} / 2 \mathbb{Z})^{n}$, one choice of $B G$ is $\left(\mathbb{R} P^{\infty}\right)^{n}$. Thus, with $\mathbb{Z} / 2 \mathbb{Z}$ coefficients,

$$
H_{G}^{*}=\mathbb{Z} / 2 \mathbb{Z}\left[x_{1}, \ldots, x_{n}\right]
$$

where $\operatorname{deg} x_{i}=1$ for all $i$.

2.2. The Thom isomorphism in $\mathbb{Z} / 2 \mathbb{Z}$-cohomology. Let $N \subset M$ be a submanifold of real codimension $k$. In ordinary cohomology (with any ring of coefficients), the Thom isomorphism is a map between the cohomology of $N$ and the compactly supported cohomology of the normal bundle $\nu N$ of $N$ in $M$. The Thom isomorphism does not preserve degree; it increases degree by $k$ :

$$
\mathbf{T}: H^{*}(N) \longrightarrow H_{c}^{*+k}(\nu N)
$$


This latter ring is identified using excision to the relative cohomology as follows:

$$
H_{c}^{*}(\nu N) \cong H^{*}(D N, S N) \cong H^{*}(M, M-N)
$$

where $D N$ and $S N$ are the disk and sphere bundles of $N$ in $M$, respectively. The class $\tau=\mathbf{T}(1)$ is called the Thom class of $\nu N$. Then $\mathbf{T}(\alpha)=p^{*} \alpha \cup \tau$, where $p^{*}$ is the map induced by the projection $p: \nu N \rightarrow N$. The class $\tau$ has the identifying property that, $\int_{\mathbb{R}^{k}} \tau=1$, where $\tau$ is restricted to the fiber of $\nu N \rightarrow N$.

In equivariant cohomology we avoid the issue of compact support by remaining in the relative cohomology ring. The following discussion is an equivariant version of well-known results found in [18] and [15]. We now restrict ourselves to the coefficient ring $\mathbb{Z} / 2 \mathbb{Z}$.

Let $G$ be a compact Lie group acting on $M$. Let $N$ be a $G$-invariant submanifold of $M$ with codimension $k$ with normal bundle $\nu N$. Then $N_{G}$ is a co-dimension $k$ submanifold of $M_{G}$. Let $\nu N_{G}$ be its normal bundle and $p: \nu N_{G} \rightarrow N_{G}$ the projection. Note that $\nu N_{G}=(\nu N)_{G}$ if $\nu N$ is identified with a $G$-invariant tubular neighborhood of $N$. Choose a $G$-invariant Riemannian metric on $\nu N_{G}$, and let $D N_{G}$ and $S N_{G}$ be the unit disk and sphere bundles, respectively. As $N$ is an oriented submanifold of $M, S N_{G}$ is an oriented sphere bundle over $N_{G}$. Consider the bundle

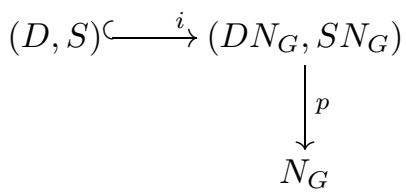

where $(D, S)$ is the fiber over any point $x \in N_{G}$ in the relative bundle $\left(D N_{G}, S N_{G}\right)$. The cohomology $H^{*}\left(D N_{G}, S N_{G}\right)$ is isomorphic to that of the relative normal bundle $H^{*}\left(\nu N_{G}, \nu N_{G}-N_{G}\right)=: H_{G}^{*}(\nu N, \nu N-N)$. There is a unique equivariant Thom class

$$
\tau_{G} \in H^{k}\left(\nu N_{G}, \nu N_{G}-N_{G}\right)=: H_{G}^{k}(\nu N, \nu N-N)
$$

(in $\mathbb{Z} / 2 \mathbb{Z}$ coefficients) such that $\tau_{G}$ restricted to the (relative) fiber over any point is the unique nonzero class in the ordinary cohomology $H^{k}(D, S)$, where $D$ and $S$ are the intersection of the fiber with the disk and sphere bundles, respectively.

In particular, the equivariant Thom isomorphism in $\mathbb{Z} / 2 \mathbb{Z}$ coefficients is the map $\mathbf{T}: H_{G}^{*}(N) \rightarrow H_{G}^{*+k}(\nu N, \nu N-N)$ obtained by the composition of $p^{*}: H_{G}^{*}(N) \rightarrow$ $H_{G}^{*}(\nu N)$ induced by the projection, and the map

$$
\cup \tau_{G}: H_{G}^{*}(\nu N) \rightarrow H_{G}^{*+k}(\nu N, \nu N-N)
$$

which multiplies any class in $H_{G}^{*}(\nu N)$ by $\tau_{G}$ using the cup product. In other words, $\mathbf{T}(\alpha)=p^{*}(\alpha) \cup \tau_{G}$.

Lemma 2.1. Let $N$ be a submanifold of $M$, where $N$ and $M$ are compact, oriented manifolds. Let $\nu N$ be the normal bundle of $N$ in $M$. Let $G$ act on $M$ fixing $N$, such that for every point $p \in \nu N-N$, there is a $\mathbb{Z} / 2 \mathbb{Z} \subset G$ acting nontrivially on p. In cohomology with $\mathbb{Z} / 2 \mathbb{Z}$ coefficients, the restriction of the equivariant Thom class $\tau_{G}$ to the submanifold $N$ is the top equivariant Stiefel-Whitney class of the bundle $\nu N$. 
Remark 2.2. The reason this theorem is so powerful is that it has the following interpretation. Let $f: N \hookrightarrow M$ be the inclusion. The composition

$$
H_{G}^{*}(N) \stackrel{\mathbf{T}}{\longrightarrow} H_{G}^{*+k}(\nu N, \nu N-N) \cong H_{G}^{*+k}(M, M-N) \stackrel{j^{*}}{\longrightarrow} H_{G}^{*+k}(M)
$$

is the pushforward $f_{*}$. Here $j^{*}$ is the map induced by the inclusion $(M, \emptyset) \subset$ $(M, M-N)$. Lemma 2.1 states that, in $\mathbb{Z} / 2 \mathbb{Z}$-cohomology, $f^{*} f_{*}(1)=w_{k}^{G}(\nu N)$, where $f^{*}$ is the pullback. In $\mathbb{Z}$-cohomology, $f^{*} f_{*}(1)=e_{G}(\nu N)$, the equivariant Euler class of the normal bundle.

Proof of Lemma 2.1. The top Stiefel-Whitney class is defined using the "squaring operation". For $a \in H_{G}^{k}(\nu N, \nu N-N ; \mathbb{Z} / 2 \mathbb{Z})$, let $S q^{k}(a)=a \cup a$. The $k^{\text {th }}$ equivariant Stiefel-Whitney class is the unique class $w_{k}^{G}(\nu N) \in H_{G}^{k}(N ; \mathbb{Z} / 2 \mathbb{Z})$ such that $\mathbf{T}\left(w_{k}^{G}(\nu N)\right)=S q^{k}\left(\tau_{G}\right)$. Thus

$$
\mathbf{T}\left(w_{k}^{G}(\nu N)\right)=p^{*}\left(w_{k}^{G}(\nu N)\right) \cup \tau_{G}=\tau_{G} \cup \tau_{G} .
$$

Since $\mathbf{T}\left(f^{*} j^{*}\left(\tau_{G}\right)\right)=p^{*}\left(f^{*} j^{*}\left(\tau_{G}\right)\right) \cup \tau_{G}=\tau_{G} \cup \tau_{G}$, and $\mathbf{T}$ is an isomorphism, it follows that $f^{*} j^{*}\left(\tau_{G}\right)=w_{k}^{G}(\nu N)$.

We show in the proof of Lemma 2.3 that, under appropriate assumptions regarding the $G$ action on $\nu N$ (an assumption implied by no 2-torsion), multiplication by the equivariant Stiefel-Whitney class is injective.

2.3. The equivariant top Stiefel-Whitney class. Suppose $M$ is compact and symplectic, equipped with an anti-symplectic involution $\sigma$. We assume that there is a compact torus $T$ acting on $M$ in a Hamiltonian fashion, and a real torus $T_{\mathbb{R}}=(\mathbb{Z} / 2 \mathbb{Z})^{n} \subset T$ acting on the real locus $Q=M^{\sigma}$. One of the critical properties of Hamiltonian actions on compact manifolds is the richness of the structure of the fixed point sets. We use the behavior of the group action on the normal bundles of these fixed sets to obtain cohomological information. The following lemma describes the cohomology locally near a fixed point set. It is crucial in making subsequent inductive arguments.

Lemma 2.3 (Atiyah-Bott). Let $E \rightarrow N$ be a rank $k$ bundle over a compact, connected oriented manifold $N$, and $G$ either a compact torus or $G=(\mathbb{Z} / 2 \mathbb{Z})^{n}$ acting on $E$ whose fixed point set is exactly $N$. Suppose also that the cohomology of $N$ has no torsion over $\mathbb{Z}$. Choose a $G$-invariant Riemannian metric and let $D E$ and $S E$ be the disk and sphere bundles, respectively, of $E$. Then the long exact sequence induced by the inclusions $(S E, \emptyset) \hookrightarrow(D E, \emptyset) \hookrightarrow(D E, S E)$ splits into short exact sequences

$$
0 \rightarrow H_{G}^{*}(D E, S E) \rightarrow H_{G}^{*}(D E) \rightarrow H_{G}^{*}(S E) \rightarrow 0,
$$

where the coefficient ring is taken to be $\mathbb{Z}$ if $G$ is a compact torus, and $\mathbb{Z} / 2 \mathbb{Z}$ for $G=(\mathbb{Z} / 2 \mathbb{Z})^{n}$.

Remark 2.4. In the case that $G=(\mathbb{Z} / 2 \mathbb{Z})^{n}$, the requirement that $G$ act on the fibers of $E$ nontrivially is weaker than the condition of no 2-torsion for $n>1$.

Remark 2.5. The condition that $N$ have no torsion is unnecessary if the coefficient ring is $\mathbb{Z} / 2 \mathbb{Z}$. 
Proof. The proof of 2.2 can be found in [4] in the case that $G=T$, a compact torus.

Suppose that $G=(\mathbb{Z} / 2 \mathbb{Z})^{n}$ and the coefficient field is $\mathbb{Z} / 2 \mathbb{Z}$. Since $N$ is fixed by $G$ we have

$$
H_{G}^{*}(N ; \mathbb{Z} / 2 \mathbb{Z})=H_{G}^{*}(p t) \otimes H^{*}(N)=\mathbb{Z} / 2 \mathbb{Z}\left[x_{1}, \ldots, x_{n}\right] \otimes H^{*}(N) .
$$

We show that the leading term of $w_{k}^{G}(E)$ in (2.1) is nonzero, where $\operatorname{deg}\left(x_{i}\right)=1$ and $k$ is the rank of $E$. As we have assumed that $N$ has no torsion, if the leading term in $H_{G}^{*}(p t) \otimes 1$ is not zero, then the equivariant Stiefel-Whitney class is not a zero-divisor.

Consider the inclusion of a point $p \hookrightarrow N$ and the induced projection

$$
H_{G}^{*}(N) \rightarrow H_{G}^{*}(p)=H_{G}^{*}
$$

We need only show that the projection of $w_{k}^{G}(E)$ is nonzero. The fiber $V$ over $p$ is a representation of $G$ which, since $G$ is simply a product of $\mathbb{Z} / 2 \mathbb{Z}$ 's, splits into real one-dimensional representations $V_{i}$ of $G$. Each of these representations is nontrivial, by our assumption that $G$ fixes exactly $N$ in the bundle $E$. The bundle $V_{i} \times{ }_{G} E G \rightarrow B G$ is the pullback of the canonical line bundle over $\mathbb{R} P^{\infty}$ under the projection $B G=\left(\mathbb{R} P^{\infty}\right)^{n} \rightarrow \mathbb{R} P^{\infty}$ to one component. This bundle has nontrivial first Stiefel-Whitney class by the axiomatic definition of these classes. Thus the projection of $w_{k}^{G}(E)$ is $w_{k}^{G}(V)=\prod_{i} w_{1}^{G}\left(V_{i}\right)$ which is nonzero.

We identify $H_{G}^{*}(D E, S E)$ with $H_{G}^{*-k}(N)$ using the Thom isomorphism, and $H_{G}^{*}(D E)$ with $H_{G}^{*}(N)$ by contraction. Then the map $H_{G}^{*}(D E, S E) \rightarrow H_{G}^{*}(D E)$ is identified with the injective map given by multiplication by the Euler class (in $\mathbb{Z}$ coefficients) or the Stiefel-Whitney class (in $\mathbb{Z} / 2 \mathbb{Z}$ coefficients). It follows that the long exact sequence

$$
\cdots \longrightarrow H_{G}^{*-1}(S E) \longrightarrow H_{G}^{*}(D E, S E) \longrightarrow H_{G}^{*}(D E) \longrightarrow H_{G}^{*}(S E) \longrightarrow \cdots
$$

splits into short exact sequences.

Another proof of this lemma can be found in [1].

\section{Morse-KirWAN THEORY FOR REAL LOCI}

We return to case where $M$ is a compact connected symplectic manifold with a Hamiltonian $T$ action with moment map $\Phi: M \rightarrow \mathfrak{t}^{*}$. Suppose also that $M$ is equipped with an anti-symplectic involution that anti-commutes with $T$. Let $Q$ be the real locus of $M$. We fix an inner product on $\mathfrak{t}^{*}$.

3.1. Morse-Kirwan functions on real loci. In this section, we will prove three important lemmas. In the first, we determine the critical sets of $\left.\|\Phi\|^{2}\right|_{Q}$ in $Q$. Duistermaat showed [1] that for any component of the moment map $\Phi^{\xi}$, if $C_{M}$ is the set of critical points for $\Phi^{\xi}$ on $M$, the set of critical points of $\left.\Phi^{\xi}\right|_{Q}$ is $\left(C_{M}\right)^{\sigma}$. We prove an analogous result for $\left.\|\Phi\|^{2}\right|_{Q}$.

Lemma 3.1. Let $C_{M}$ be the set of critical points of the function $\|\Phi\|^{2}: M \rightarrow \mathbb{R}$, and let $C_{Q}$ be the set of critical points of $\left\|\left.\Phi\right|_{Q}\right\|^{2}: Q \rightarrow \mathbb{R}$. Then $C_{M}$ is preserved by $\sigma$, and $C_{M}^{\sigma}=C_{Q}$.

Proof. Recall that $Q$ is the fixed point set of the involution $\sigma$, and that $\sigma$ commutes with the moment map $\Phi$. For any point $p \in Q$ we have $T_{p} Q=\operatorname{ker}\left(d \sigma_{p}-I\right)=$ $\left\{v+d \sigma_{p}(v) \mid v \in T_{p} M\right\}$. 
Suppose $p$ is critical for $\|\Phi\|^{2}$. The involution $\sigma$ induces an isomorphism of tangent spaces. So $\Phi \circ \sigma=\Phi$ implies that $\sigma(p)$ is critical for $\|\Phi\|^{2}$. Thus $C_{M}$ is preserved by $\sigma$.

Suppose that a $p \in Q$ is critical for $\|\Phi\|^{2}$. Then clearly $p$ is critical for $\left\|\left.\Phi\right|_{Q}\right\|^{2}$, so $C_{M}^{\sigma} \subseteq C_{Q}$.

Now suppose that $p \in Q$ is critical for $\left\|\left.\Phi\right|_{Q}\right\|^{2}$. Then for all $v \in T_{p} M$,

$$
\begin{aligned}
0 & =d\left\|\left.\Phi\right|_{Q}\right\|_{p}^{2}\left(v+d \sigma_{p}(v)\right) \\
& =2 \Phi(p) \cdot d\left(\left.\Phi\right|_{Q}\right)_{p}\left(v+d \sigma_{p}(v)\right) \\
& =2 \Phi(p) \cdot\left[d(\Phi)_{p}(v)+d \Phi_{p}\left(d \sigma_{p}(v)\right)\right] \\
& =2 \Phi(p) \cdot\left[d(\Phi)_{p}(v)+d(\Phi \circ \sigma)_{p}(v)\right] \\
& =2 \Phi(p) \cdot\left[d(\Phi)_{p}(v)+d(\Phi)_{p}(v)\right] \\
& =4 \Phi(p) \cdot d \Phi_{p}(v)=2 d\|\Phi\|_{p}^{2}(v),
\end{aligned}
$$

so $p$ is critical for $\|\Phi\|^{2}$ as well.

In the next two lemmas, we show that the functions $\left.\Phi^{\xi}\right|_{Q}$ and $\left.\|\Phi\|^{2}\right|_{Q}$ are MorseKirwan functions on $Q$, when $Q$ contains no 2-torsion points. As before, $M$ is compact, connected, and symplectic with a Hamiltonian torus action and involution $\sigma$ fixing the real locus $Q$. We suppose also that $Q$ contains no 2-torsion points. The following two lemmas are real locus versions of results of Kirwan [14. The proof of the first lemma is nearly identical to that given by Tolman and Weitsman in [20. The only differences that occur are a result of the possibility of 2-torsion points. The proof of the second lemma contains some additional analysis concerning critical sets. We include the proofs here for completeness.

Lemma 3.2. Choose an invariant Riemannian metric on $M$. Given any $\xi \in \mathfrak{t}$, define $f=\left.\Phi^{\xi}\right|_{Q}$. Let $C$ be a critical set of index $\lambda$ for $f$, and assume that $C$ is the only critical set in the preimage of an $\varepsilon$-neighborhood around $f(C)$, for some $\varepsilon>0$. Define

$$
Q^{ \pm}=f^{-1}((-\infty, f(C) \pm \varepsilon)) .
$$

Then the long exact sequence of the pair $\left(Q^{+}, Q^{-}\right)$splits into short exact sequences in equivariant cohomology, with $\mathbb{Z} / 2 \mathbb{Z}$ coefficients:

$$
0 \rightarrow H_{T_{\mathbb{R}}}^{*}\left(Q^{+}, Q^{-}\right) \rightarrow H_{T_{\mathbb{R}}}^{*}\left(Q^{+}\right) \rightarrow H_{T_{\mathbb{R}}}^{*}\left(Q^{-}\right) \rightarrow 0 .
$$

Proof. The function $f$ is Morse-Bott at every connected component of the critical set. When $\xi$ is generic, the critical sets are precisely the connected components of the fixed points. When $\xi$ is not generic, then the critical sets are connected components of $\left(M^{K}\right)^{\sigma}$, where $K \subseteq T$ is some closed subtorus of $T$. Since the action is smooth, these sets are submanifolds. The negative normal bundle to $C$ is oriented, except at the minimum, where the negative normal bundle is zero. Consider the long exact sequence in equivariant cohomology with $\mathbb{Z} / 2 \mathbb{Z}$ coefficients,

$$
\cdots \rightarrow H_{T_{\mathbb{R}}}^{*}\left(Q^{+}, Q^{-}\right) \rightarrow H_{T_{\mathbb{R}}}^{*}\left(Q^{+}\right) \rightarrow H_{T_{\mathbb{R}}}^{*}\left(Q^{-}\right) \rightarrow H_{T_{\mathbb{R}}}^{*+1}\left(Q^{+}, Q^{-}\right) \rightarrow \cdots .
$$

Let $c=f(C)$ be a nonminimal critical value corresponding to $C$. By assumption, $c$ is the only critical value in the interval $[c-\varepsilon, c+\varepsilon]$. Denote the negative disc and sphere bundles to $C$ in $Q$ by $D_{c}$ and $S_{c}$ respectively. We let $\lambda$ denote the Morse 
index of $C$ in $Q$. Following an identical argument to [20, Proof of Proposition 2.1], we obtain a commutative diagram, with $\mathbb{Z} / 2 \mathbb{Z}$ coefficients,

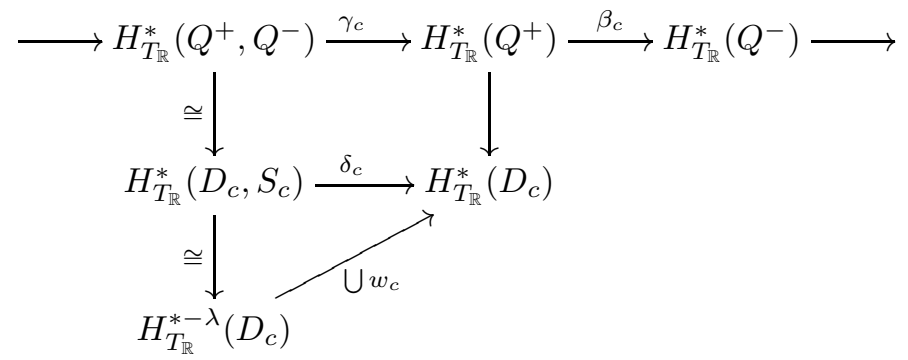

where $w_{c}=w_{k}\left(D_{c}\right)$ is the equivariant top Stiefel-Whitney class of the bundle $D_{c} \rightarrow C$. The left-most vertical arrows in the diagram are excision and the Thom isomorphism with $\mathbb{Z} / 2 \mathbb{Z}$ coefficients. Remark 2.2 ensures that the diagonal arrow is indeed the cup product with $w_{c}$. Because there are no 2-torsion points in $C \subseteq Q$, $T_{\mathbb{R}}$ fixes no sub-bundle of the negative normal bundle. Lemma 2.3 ensures that the cup product map $\bigcup w_{c}$ is injective, and so $\delta_{c}$ and $\gamma_{c}$ must also be injective. Thus, the long exact sequence splits.

If $a=f(C)$ is the minimum critical value, the spaces $Q^{+}$and $C$ are equivariantly homotopic, and $Q^{-}$is empty. Thus, $H_{T_{\mathbb{R}}}^{*}\left(Q^{-}\right)=0$ and $H_{T_{\mathbb{R}}}^{*}\left(Q^{+}\right) \cong H_{T_{\mathbb{R}}}^{*}(C)$. Therefore, the sequence splits in this case as well. This completes the proof of the lemma.

Lemma 3.3. Choose an invariant Riemannian metric on $M$. Given $a \in \mathfrak{t}^{*}$, let $f_{a}(x)=\langle\Phi(x)-a, \Phi(x)-a\rangle=\|\Phi(x)-a\|^{2}$. Let $X \subset M$ be a critical set for $f_{a}$, and assume that $X$ is the only critical set in the preimage of an $\varepsilon$-neighborhood around $f_{a}(X)$, for some $\varepsilon>0$. Define

$$
Q_{a}^{ \pm}=f_{a}^{-1}\left(\left(-\infty, f_{a}(X) \pm \varepsilon\right)\right) \cap Q .
$$

Then the long exact sequences of the pair $\left(Q_{a}^{+}, Q_{a}^{-}\right)$split into short exact sequences in equivariant cohomology, with $\mathbb{Z} / 2 \mathbb{Z}$ coefficients:

$$
0 \rightarrow H_{T_{\mathbb{R}}}^{*}\left(Q_{a}^{+}, Q_{a}^{-}\right) \rightarrow H_{T_{\mathbb{R}}}^{*}\left(Q_{a}^{+}\right) \rightarrow H_{T_{\mathbb{R}}}^{*}\left(Q_{a}^{-}\right) \rightarrow 0 .
$$

Proof. Suppose $X$ is not the critical set at the 0-level set. While the function $f_{a}$ may not be Morse-Bott at $X$, Kirwan proves 14 , that the critical sets behave on a cohomological level as if they were nondegenerate. There is a smooth stratification $\left\{S_{\alpha}, \alpha \in B\right\}$ of $M$, where $B$ is an index set of vectors in $\mathfrak{t}^{*}$, such that, for some $\beta \in B$, the stratum $S_{\beta}$ contains precisely those $x \in M$ such that the limit of the path of steepest descent for $f_{a}$ from $x$ is in $X$. Kirwan proves that the inclusion $X \subset S_{\beta}$ is an equivalence of $T$-equivariant cohomology. Furthermore, close to $X$, $S_{\beta}$ coincides with a $T$-invariant submanifold $\Sigma$ of a neighborhood of $X$, and $\Sigma$ has a well-defined orientable normal bundle.

Let $X^{\sigma}$ be the fixed point set of the involution $\sigma$ of $X$. All critical sets of $f_{a}$ restricted to $Q$ will be of the form $X^{\sigma}$, by Lemma 3.1. Then $X^{\sigma}$ is the only critical set of $\left.f_{a}\right|_{Q}$ in $Q_{a}^{+}$and not in $Q_{a}^{-}$. We assume $X^{\sigma}$ is connected, for each component can be treated individually by a direct sum argument in the following discussion. $X^{\sigma}$ lies in $\Sigma^{\sigma}$, the submanifold of a neighborhood of $X^{\sigma}$ in $Q$ formed by the fixed point set of $\sigma$ on $\Sigma$ ( $\Sigma^{\sigma}$ is smooth since $\sigma$ is a smooth action on $\left.\Sigma\right)$. Furthermore, 
$\Sigma^{\sigma}$ has a well-defined oriented normal bundle in $Q, \Sigma^{\sigma}$ coincides with $S_{\beta}^{\sigma}$ close to $X^{\sigma}$, and $X^{\sigma} \subset S^{\sigma}$ is an equivalence in $T$-equivariant cohomology by [6].

Consider the long exact sequence in equivariant cohomology with $\mathbb{Z} / 2 \mathbb{Z}$ coefficients,

$$
\cdots \rightarrow H_{T_{\mathbb{R}}}^{*}\left(Q_{a}^{+}, Q_{a}^{-}\right) \rightarrow H_{T_{\mathbb{R}}}^{*}\left(Q_{a}^{+}\right) \rightarrow H_{T_{\mathbb{R}}}^{*}\left(Q_{a}^{-}\right) \rightarrow H_{T_{\mathbb{R}}}^{*+1}\left(Q_{a}^{+}, Q_{a}^{-}\right) \rightarrow \cdots
$$

Except at the minimum, there is a commutative diagram of the form (3.1). The 2 -torsion hypothesis guarantees that $T_{\mathbb{R}}$ acts nontrivially on the negative normal bundle to $\Sigma^{\sigma}$, and that the cup product with the equivariant Stiefel-Whitney class is an injection. By Kirwan's work (extended to the real case as above) the negative normal bundle to $\Sigma^{\sigma}$ plays the role (cohomologically) that the negative normal bundle to the critical set plays in the case that the critical set is itself a smooth manifold.

Finally, at the minimum $f_{a}^{-1}(0)$, the spaces $Q_{a}^{+}$and $X^{\sigma}$ are equivariantly homotopic, and $Q_{a}^{-}$is empty. Thus, $H_{T_{\mathbb{R}}}^{*}\left(Q_{a}^{-}\right)=0$ and $H_{T_{\mathbb{R}}}^{*}\left(Q_{a}^{+}\right) \cong H_{T_{\mathbb{R}}}^{*}\left(X^{\sigma}\right)$. The lemma now follows.

3.2. Kirwan's injectivity theorem for real loci. Let $M$ be a compact symplectic manifold with Hamiltonian $T$-action with moment map $\Phi$. Let $M^{T}$ denote the set of fixed points of $M$. Kirwan's injectivity theorem [14] states that the inclusion

$$
i: M^{T} \hookrightarrow M
$$

induces an injection in equivariant cohomology

$$
i^{*}: H_{T}^{*}(M) \hookrightarrow H_{T}^{*}\left(M^{T}\right) .
$$

The real locus analogue is proved in [6] using algebraic techniques. Schmid hints that there is a Morse-Kirwan theoretic proof in [17, but does not provide the details.

Theorem 3.4 ([6], 17). Let $M$ be a compact symplectic manifold with Hamiltonian $T$-action with moment map $\Phi$. Let $Q$ be the real locus of $M$ and let $F=Q^{T_{R}}$ be the fixed points of $T_{\mathbb{R}}$ in $Q$, with natural inclusion map $i: F \hookrightarrow Q$. Assume that $F$ contains no 2 -torsion points. Then the pullback in equivariant $\mathbb{Z} / 2 \mathbb{Z}$-cohomology

$$
i^{*}: H_{T_{\mathbb{R}}}^{*}(Q) \rightarrow H_{T_{\mathbb{R}}}^{*}(F)
$$

is injective.

Proof. Choose an element $\xi \in \mathfrak{t}$ such that the critical set of $\Phi^{\xi}$ is precisely $F$. Order the critical values of $f=\Phi^{\xi}$ as $c_{1}<\cdots<c_{N}$, and let $F_{i}$ be the critical points with $f\left(F_{i}\right)=c_{i}$. Denote

$$
Q_{i}^{ \pm}=f^{-1}\left(\left(-\infty, c_{i} \pm \varepsilon\right)\right) .
$$

The theorem holds for $Q_{1}^{-}$because this set is empty. We proceed by induction. Suppose that

$$
H_{T_{\mathbb{R}}}^{*}\left(Q_{i}^{-}\right) \hookrightarrow H_{T_{\mathbb{R}}}^{*}\left(Q_{i}^{-} \cap F\right)
$$

is an injection. We need to apply Lemma 3.2, at the critical sets of $\Phi^{\xi}$, which are precisely the fixed point components of $F$. Thus, since $F$ contains no 2-torsion points, we conclude that there is a short exact sequence

$$
0 \rightarrow H_{T_{\mathbb{R}}}^{*}\left(Q_{i}^{+}, Q_{i}^{-}\right) \rightarrow H_{T_{\mathbb{R}}}^{*}\left(Q_{i}^{+}\right) \rightarrow H_{T_{\mathbb{R}}}^{*}\left(Q_{i}^{-}\right) \rightarrow 0 .
$$


Let $i_{ \pm}^{*}$ be the inclusion $Q_{i}^{ \pm} \cap F \hookrightarrow Q_{i}^{ \pm}$. Then we have a commutative diagram, with $\mathbb{Z} / 2 \mathbb{Z}$ coefficients,

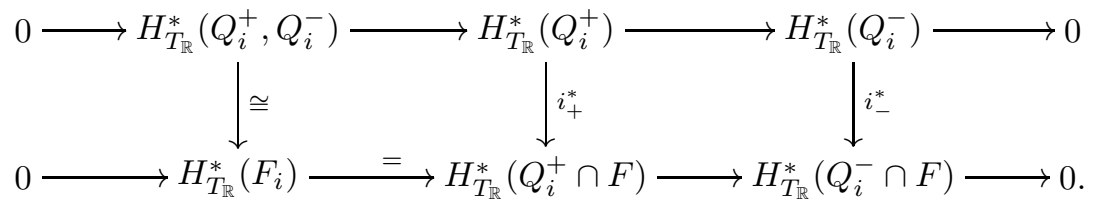

The map $i_{-}^{*}$ is an injection by the inductive hypothesis. A diagram chase shows that $i_{+}^{*}$ is an injection. Finally, notice that $Q_{i}^{+}$is equivariantly homotopy equivalent to $Q_{i+1}^{-}$. This completes the proof.

\section{Proving surjectivity (Theorem 1)}

4.1. Reductions of real loci. Suppose that $\mu$ is a regular value of $\Phi$, and that $T$ acts freely on $\Phi^{-1}(\mu)$. Then $M_{r e d}=M / / T(\mu)$ is a symplectic manifold with a canonical symplectic form $\omega_{\text {red }}$. Notice that $\Phi$ is $\sigma$-invariant: $\Phi(\sigma(x))=\Phi(x)$. Thus, $\sigma$ acts on the level sets $\Phi^{-1}(\mu)$, taking $T$-orbits to $T$-orbits. Therefore, there is an induced involution $\sigma_{\text {red }}$ on $M_{\text {red }}$. There are natural maps

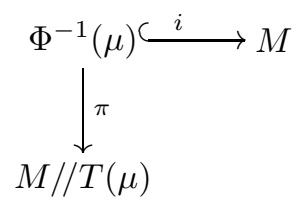

The symplectic form $\omega_{\text {red }}$ on $M / / T(\mu)$ satisfies the property that $\pi^{*} \omega_{r e d}=i^{*} \omega$. It is immediate that $\sigma$ also satisfies $\pi^{*} \sigma_{\text {red }}=i^{*} \sigma$.

Proposition 4.1. The involution $\sigma_{\text {red }}$ on $M / / T(\mu)$ is anti-symplectic with respect to $\omega_{\text {red }}$.

Proof. Notice first that

$$
\begin{aligned}
i^{*}\left(\sigma^{*} \omega\right) & =-i^{*} \omega, \quad \text { since } \sigma^{*} \omega=-\omega \\
& =-\pi^{*} \omega_{\text {red }} \\
& =\pi^{*}\left(-\omega_{\text {red }}\right) .
\end{aligned}
$$

But now we also notice that

$$
\begin{aligned}
i^{*}\left(\sigma^{*} \omega\right) & =\left(i^{*} \sigma\right)^{*}\left(i^{*}(\omega)\right), \quad \text { since } \Phi \text { is } \sigma \text {-invariant } \\
& =\left(\pi^{*} \sigma_{\text {red }}\right)^{*}\left(\pi^{*}\left(\omega_{\text {red }}\right)\right) \\
& =\pi^{*}\left(\sigma_{\text {red }}^{*} \omega_{\text {red }}\right) .
\end{aligned}
$$

Thus $\pi^{*}\left(-\omega_{\text {red }}\right)=\pi^{*}\left(\sigma_{\text {red }}^{*} \omega_{\text {red }}\right)$. Finally, we notice that $\pi^{*}$ is an injection, completing the proof.

Corollary 4.2. The real locus $(M / / T(\mu))^{\sigma_{\text {red }}}$ of $M / / T(\mu)$ is a Lagrangian submanifold.

Proof. This follows immediately from the proposition, as Duistermaat has shown in [11] that the real locus of any symplectic manifold with any anti-symplectic involution is Lagrangian. 
The torus $T$ acts freely on $\Phi^{-1}(\mu)$, so the subset $T_{\mathbb{R}}$ of order two elements acts freely on $\left(\left.\Phi\right|_{Q}\right)^{-1}(\mu)$. Thus the reduction of the real locus is well defined:

$$
Q / / T_{\mathbb{R}}(\mu):=\left(\left(\left.\Phi\right|_{Q}\right)^{-1}(\mu)\right) / T_{\mathbb{R}} .
$$

Proposition 4.3. Let $M$ be a compact symplectic manifold, and suppose $T$ acts on $M$ in a Hamiltonian fashion. Suppose $M$ has real locus $Q$. Let $\mu \in \mathfrak{t}^{*}$ be a regular value of the moment map such that $T$ acts freely on $\Phi^{-1}(\mu)$. Then

$$
(M / / T(\mu))^{\sigma_{\text {red }}}=Q / / T_{\mathbb{R}}(\mu) .
$$

Proof. The symplectic reduction $M / / T(\mu)$ consists of $T$-orbits with moment image $\mu$. The points of $(M / / T(\mu))^{\sigma_{\text {red }}}$ are those orbits which are fixed by $\sigma$. On the other hand, the reduction $Q / / T_{\mathbb{R}}(\mu)$ consists of $T_{\mathbb{R}}$-orbits in $Q$ with moment image $\mu$.

Given a $T$-orbit $T \cdot y$ fixed by $\sigma$, there is a $T_{\mathbb{R}}$-orbit in $T \cdot y$ that is fixed pointwise by $\sigma$, for suppose that $\sigma(y)=t \cdot y$ for some $t \in T$. Then

$$
x=\sigma(\sqrt{t} \cdot y)=\sqrt{t} \cdot y
$$

is fixed by $\sigma$, and moreover, $T_{\mathbb{R}} \cdot x$ is the orbit we seek. In particular, we can view

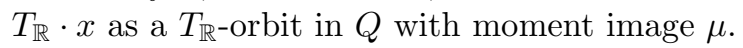

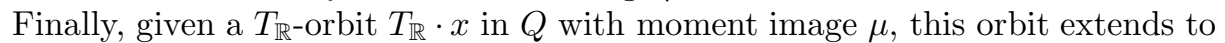
$T \cdot x$, a $T$-orbit fixed by $\sigma$ in $M$. We claim that this is the only $T_{\mathbb{R}}$-orbit extending

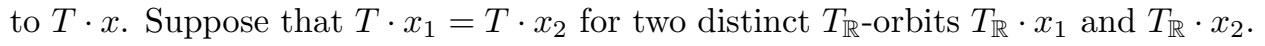
Then there is a $t \in T$ such that $x_{1}=t \cdot x_{2}$. But then, $\sigma\left(x_{1}\right)=\sigma\left(t \cdot x_{2}\right)$. Thus, $x_{1}=t^{-1} \cdot x_{2}$. But now, because $T$ acts freely on $\Phi^{-1}(\mu)$, the element $t$ must be in $T_{\mathbb{R}}$, so $T_{\mathbb{R}} \cdot x_{1}=T_{\mathbb{R}} \cdot x_{2}$, completing the proof.

4.2. The proof of surjectivity. In this section, we will prove that there is a surjection from the $T_{\mathbb{R}}$-equivariant cohomology of $Q$ onto the ordinary cohomology of $Q / / T_{\mathbb{R}}(\mu)$. We will first show this for $\mu=0$, and we can then deduce the theorem for all other values $\mu$ by shifting the moment map $\Phi$ appropriately. We will use the function $f=\|\Phi\|^{2}$ as a Morse-Kirwan function on $Q$ and apply Lemma 2.3 inductively to show that $H_{T_{\mathbb{R}}}^{*}(Q) \rightarrow H^{*}\left(Q / / T_{\mathbb{R}}(0)\right)$ with $\mathbb{Z} / 2 \mathbb{Z}$ coefficients is a surjective map.

Suppose a torus $T$ acts on $M$ in a Hamiltonian fashion. Let 0 be a regular value of the moment map $\Phi$, and assume that $T$ acts freely on $\Phi^{-1}(0)$. Suppose further that $Q$ is the real locus of $M$ and that $Q$ contains no 2-torsion points. Then Theorem 1 states that the map with $\mathbb{Z} / 2 \mathbb{Z}$ coefficients

$$
H_{T_{\mathbb{R}}}^{*}(Q) \rightarrow H^{*}\left(Q / / T_{\mathbb{R}}(0)\right)
$$

induced by inclusion is a surjection. We prove this now.

Proof of Theorem 1] Let $f_{0}=\left.\|\Phi\|^{2}\right|_{Q}$, and let $c_{0}<c_{1}<\cdots<c_{N}$ be the finitely many critical values of $f_{0}$, where $c_{0}=0$. Choose $\varepsilon>0$ so that $c_{i}+\varepsilon<c_{i+1}$ and $c_{i}-\varepsilon>c_{i-1}$ for all $i$. Let

$$
Q_{i}^{ \pm}=f_{0}^{-1}\left(\left(-\infty, c_{i} \pm \varepsilon\right)\right)
$$

The set $Q_{0}^{-}$is empty, while $Q_{0}^{+}$is equivariantly homotopy equivalent to $f_{0}^{-1}(0)$. The free $T_{\mathbb{R}}$ action implies

$$
H_{T_{\mathbb{R}}}^{*}\left(Q_{0}^{+}\right)=H^{*}\left(Q / / T_{\mathbb{R}}(0)\right),
$$


in $\mathbb{Z} / 2 \mathbb{Z}$ coefficients. We note that $Q_{i}^{+}$is equivariantly homotopy equivalent to $Q_{i+1}^{-}$and $Q_{N}^{+}=Q$. As $Q$ has no 2-torsion points, we apply Lemma 3.3 to $Q_{i}^{+}$and $Q_{i}^{-}$to obtain a short exact sequence with $\mathbb{Z} / 2 \mathbb{Z}$ coefficients

$$
0 \rightarrow H_{T_{\mathbb{R}}}^{*-\lambda_{i}}\left(f_{0}^{-1}\left(c_{i}\right)\right) \rightarrow H_{T_{\mathbb{R}}}^{*}\left(Q_{i}^{+}\right) \rightarrow H_{T_{\mathbb{R}}}^{*}\left(Q_{i}^{-}\right) \rightarrow 0 .
$$

Thus, we have a surjection $H_{T_{\mathbb{R}}}^{*}\left(Q_{i}^{+}\right) \rightarrow H_{T_{\mathbb{R}}}^{*}\left(Q_{i-1}^{+}\right)$over $\mathbb{Z} / 2 \mathbb{Z}$, because $Q_{i}^{-}$is equivariantly homotopy equivalent to $Q_{i-1}^{+}$. Over all $i$ we obtain a sequence of surjections in equivariant cohomology with $\mathbb{Z} / 2 \mathbb{Z}$ coefficients

$$
\begin{gathered}
H_{T_{\mathbb{R}}}^{*}(Q)=H_{T_{\mathbb{R}}}^{*}\left(Q_{N}^{+}\right) \rightarrow H_{T_{\mathbb{R}}}^{*}\left(Q_{N-1}^{+}\right) \rightarrow \cdots \\
\cdots \rightarrow H_{T_{\mathbb{R}}}^{*}\left(Q_{1}^{+}\right) \rightarrow H_{T_{\mathbb{R}}}^{*}\left(Q_{0}^{+}\right)=H^{*}\left(Q / / T_{\mathbb{R}}(0)\right),
\end{gathered}
$$

induced by inclusions $Q_{i}^{+} \hookrightarrow Q_{i+1}^{+}$. The composition is the Kirwan map (4.1).

We have only proved the theorem for the regular value $\mu=0$. Suppose $\mu$ is some other regular value of $\Phi$ such that $T$ acts freely on $\Phi^{-1}(\mu)$. Since $\Phi$ is determined only up to its constant term, we may choose a new moment map $\Psi=\Phi-\mu$. This new moment map will have $\Psi^{-1}(0)=\Phi^{-1}(\mu)$, and so we may apply the above argument to $\Psi$ to prove that there is a surjection

$$
\kappa_{\mathbb{R}}: H_{T_{\mathbb{R}}}^{*}(Q) \rightarrow H^{*}\left(Q / / T_{\mathbb{R}}(\mu)\right) .
$$

\section{The kernel of the real Kirwan map (Theorem 2)}

We now compute the kernel of the real Kirwan map, $\kappa_{\mathbb{R}}$ (Theorem 2). The technique for real loci is identical to the symplectic analogue. However, we must impose some additional requirements on our real locus. First, we require injectivity of the equivariant cohomology of the real locus to the equivariant cohomology of its fixed point set (Theorem 3.4). Second, we require that the multiplication by the top Stiefel-Whitney class of the negative normal bundles to certain functions be nonzero. This follows from the proof of Lemma 2.3 . Here the $(\mathbb{Z} / 2 \mathbb{Z})^{n}$-equivariant Stiefel-Whitney class plays the role that the equivariant Euler class does in [19].

Following [19] but restricting our attention to the real locus, we define

$$
Q_{\xi}=\{p \in Q \mid\langle\Phi(p), \xi\rangle \leq 0\} .
$$

for any $\xi \in \mathfrak{t}$, and

$$
K_{\xi}=\left\{\alpha \in H_{T_{\mathbb{R}}}^{*}(Q ; \mathbb{Z} / 2 \mathbb{Z})|\alpha|_{F \cap Q_{\xi}}=0\right\},
$$

where $F=Q^{T_{\mathbb{R}}}$. Finally, define the ideal

$$
K_{\mathbb{R}}=\left\langle\sum_{\xi \in \mathfrak{t}} K_{\xi}\right\rangle .
$$

Theorem 2 states that $K_{\mathbb{R}}$ is the kernel of $\kappa_{\mathbb{R}}$.

Proof of Theorem Q First, we show that $K_{\mathbb{R}} \subseteq \operatorname{ker}\left(\kappa_{\mathbb{R}}\right)$. Let $\alpha \in K_{\xi}$ be a class in $K_{\mathbb{R}}$ for some $\xi \in \mathfrak{t}$. The real-valued function $f=\left.\Phi^{\xi}\right|_{Q}$ is a Morse-Kirwan function on $Q$, in the sense of Lemma 3.2. Let $d_{1}<\cdots<d_{n}$ be the critical values of $f$, and let $i$ be such that $d_{i}<0<d_{i+1}$. Applying Lemma 3.2 inductively to the 
function $f$, we see that $\left.\alpha\right|_{Q_{\xi}}=0$. But $Q_{\xi}$ is homotopy equivalent to $Q_{i+1}^{-}$, where $Q_{i+1}^{-}=f^{-1}\left(-\infty, d_{i+1}-\epsilon\right)$ for sufficiently small $\epsilon$. Then $\left.\Phi\right|_{Q} ^{-1}(0) \subset f^{-1}(0)$ implies

$$
\left.\alpha\right|_{\left.\Phi\right|_{Q} ^{-1}(0)}=\left.\alpha\right|_{f^{-1}(0)}=0 .
$$

That is, $\alpha \in \operatorname{ker}\left(\kappa_{\mathbb{R}}\right)$.

Next, following methods introduced in [19, we show that $\operatorname{ker}\left(\kappa_{\mathbb{R}}\right) \subseteq K_{\mathbb{R}}$. A class $\alpha \in \operatorname{ker}\left(\kappa_{\mathbb{R}}\right)$ implies that $\left.\alpha\right|_{\Phi^{-1}(0)^{\sigma}}=0$. By the injectivity on the real locus (Theorem 3.4), it suffices to find $\beta \in K_{\mathbb{R}}$ such that $\left.\beta\right|_{F_{i}}=\left.\alpha\right|_{F_{i}}$ for all components $F_{i}$ of the fixed point set $Q^{T_{\mathbb{R}}}$.

Let $f_{0}=\left.\|\Phi\|^{2}\right|_{Q}$, and order the critical sets $C_{0}, \ldots, C_{N}$ of $f_{0}$ with $f_{0}\left(C_{i}\right)=c_{i}$ so that the critical values are $0=c_{0}<c_{1}<\cdots<c_{N}$. Note that $d\|\Phi\|^{2}=d\|\|^{2} \circ d \Phi$ and so we find the critical sets directly. For any $x \in Q$ and $v \in T_{x} Q$ we have

$$
\begin{aligned}
d\|\Phi\|_{x}^{2}(v) & =d\|\|_{\Phi(x)}^{2} \circ d \Phi_{x}(v) \\
& =2 \Phi(x) \cdot d \Phi_{x}(v) .
\end{aligned}
$$

In particular, critical points consist of $x \in \Phi^{-1}(0)$, as well as any points $x \in Q$ such that $d\left(\left.\|\Phi\|^{2}\right|_{Q}\right)_{x}(v)=0$ for all $v \in T_{x} Q$, or $d\left(\left.\|\Phi\|^{2}\right|_{Q}\right)_{x}(v)$ is perpendicular to $\Phi(x)$ for all $v \in T_{x} Q$. Since the differential of $\Phi$ is 0 at fixed points, the fixed point set $Q^{T_{\mathbb{R}}}$ is critical for $f_{0}$. Note that $\left.\alpha\right|_{C_{0}}=0$.

Now assume that $\left.\alpha\right|_{C_{i}}=0$ for all $i<p$ and $\left.\alpha\right|_{C_{p}} \neq 0$. Applying Lemma 3.3 to $f_{0}$, we see that $\left.\alpha\right|_{C_{p}}=m w_{k}$, where $m w_{k} \in H_{T_{\mathbb{R}}}^{*}\left(C_{p}\right)$ is some multiple of the equivariant top Stiefel-Whitney class $w_{k}$ of the negative normal bundle $\nu_{f_{0}}^{-} C_{p}$ to $C_{p}$ for $f_{0}$.

Suppose $\left.\alpha\right|_{C_{i}}=0, i=1, \ldots, p-1$. We find $\beta_{p} \in K_{\mathbb{R}}$ such that $\left.\alpha\right|_{C_{i}}=\left.\left(\beta_{p}\right)\right|_{C_{i}}$, $i=1, \ldots, p$. Then $\left.\left(\alpha-\beta_{p}\right)\right|_{C_{i}}=0$ for $i=1, \ldots, p$ and we apply the argument inductively to find $\alpha=\sum_{k=p}^{N} \beta_{k} \in K_{\mathbb{R}}$. Let

$$
\mathcal{S}=\left\{F \in Q_{c c}^{T_{\mathbb{R}}} \mid \Phi(F) \cdot \Phi\left(C_{p}\right)<0\right\}
$$

where $Q_{c c}^{T_{\mathbb{R}}}$ denotes the connected components of the fixed point set. Note that if $\beta_{p} \in H_{T_{\mathbb{R}}}^{*}(Q)$ and $\left.\beta_{p}\right|_{\mathcal{S}}=0$, then $\beta_{p} \in K_{\mathbb{R}}$.

The class $\beta_{p}$ is constructed by finding a new Morse-Bott-Kirwan function $h$ on $Q$ such that:

(1) $Q^{T_{\mathbb{R}}}$ is critical for $h$.

(2) $C_{p}$ is critical for $h$, and the negative normal bundle $\nu_{h}^{-} C_{p}$ equals $\nu_{f_{0}}^{-} C_{p}$.

(3) $h\left(C_{i}\right)<h\left(C_{p}\right)$ for all $i=1, \ldots, p-1$.

(4) $h(F)<h\left(C_{p}\right)$ implies $\Phi(F) \cdot \Phi\left(C_{p}\right)<0$ for any component $F$ of $Q^{T_{\mathbb{R}}}$.

Provided such a function $h$ exists, we construct $\beta_{p}$ as follows. Let $\bar{C}_{0}, \ldots, \bar{C}_{M}$ be critical sets of $h$ such that $h\left(\bar{C}_{0}\right)<\cdots<h\left(\bar{C}_{M}\right)$. Since $C_{p}$ is critical, we let $\left.\left(\beta_{p}\right)\right|_{\bar{C}_{i}}=0$ for all $\bar{C}_{i}$ with $h\left(\bar{C}_{i}\right)<h\left(C_{p}\right)$. By Theorem 3.3 we may choose

$$
\begin{aligned}
\left.\beta_{p}\right|_{C_{p}} & =m w_{k}\left(\nu_{h}^{-} C_{p}\right) \\
& =m w_{k}\left(\nu_{f_{0}}^{-} C_{p}\right)=m w_{k} \text { by condition (2). }
\end{aligned}
$$

By condition (1) we have $\left.\beta_{p}\right|_{F}=0$ for all connected components $F$ in $Q^{T_{\mathbb{R}}}$ such that $h(F)<h\left(C_{p}\right)$. It then follows by (3) that $\left.\beta_{p}\right|_{C_{i}}=0$ for all $i=1, \ldots, p-1$ by the argument at the beginning of this proof. Lastly, we note that by condition (4), $\beta_{p} \in K_{\mathbb{R}}$. 
It is left to prove such a function exists. This is achieved by perturbing $f_{0}$ into a family of functions parametrized by $\lambda \in \mathbb{R}^{+}$, all of which are equivariantly perfect, and then we apply Lemma 3.3 to an appropriate perturbation to find $\beta$. Define

$$
f_{\lambda a}=\left\|\left.\Phi\right|_{Q}+\lambda a\right\|^{2}
$$

where $a=c_{p}$. Note that for all $x \in Q$ and $v \in T_{x} Q$,

$$
\begin{aligned}
\left.d\left(f_{\lambda a}\right)\right|_{x}(v) & =\left.\left.d\left(\|\|^{2}\right)\right|_{\left.\Phi\right|_{Q}(x)+\lambda a} \circ d\left(\left.\Phi\right|_{Q}+\lambda a\right)\right|_{x}(v) \\
& =\left.2\left(\left.\Phi\right|_{Q}(x)+\lambda a\right) \cdot d\left(\left.\Phi\right|_{Q}+\lambda a\right)\right|_{x}(v) \\
& =\left.2\left(\left.\Phi\right|_{Q}(x)+\lambda a\right) \cdot d\left(\left.\Phi\right|_{Q}\right)\right|_{x}(v)
\end{aligned}
$$

Thus $f_{\lambda a}$ is singular on $Q$ exactly when $x \in \Phi^{-1}(-\lambda a)$, when $\left.d\left(\left.\Phi\right|_{Q}\right)\right|_{x}(v)=0$ or when $d\left(\left.\Phi\right|_{Q}\right)_{x}(v)$ is perpendicular to $\left.\Phi\right|_{Q}(x)+\lambda a$. In particular, $f_{\lambda a}$ is singular on $Q^{T_{\mathbb{R}}}$, satisfying condition (1). We note also that $C_{p}$ is critical for $f_{\lambda a}$ (condition (2)), since for $x \in C_{p}$ and $v \in T_{x} Q$,

$$
\begin{aligned}
\left.d\left(f_{\lambda a}\right)\right|_{x}(v) & =\left(\left.\Phi\right|_{Q}(x)+\lambda a\right) \cdot d\left(\left.\Phi\right|_{Q}\right)_{x}(v) \\
& =(1+\lambda) a \cdot d\left(\left.\Phi\right|_{Q}\right)_{x}(v) \text { since } \Phi(x)=\Phi\left(C_{p}\right)=c_{p}=a \\
& =\left.(1+\lambda) \frac{1}{2} d\left(\left.\|\Phi\|^{2}\right|_{Q}\right)\right|_{x}(v) \\
& =0, \text { since } C_{p} \text { is critical for }\|\Phi\|^{2} .
\end{aligned}
$$

This argument also shows that condition (3) holds: the normal bundles $\nu_{f_{0}}^{-} C_{p}$ and $\nu_{f_{\lambda a}}^{-} C_{p}$ are equal, since $\left.d\left(f_{\lambda a}\right)\right|_{x}(v)<0$ if and only if $d\left(f_{0}\right)_{x}(v)<0$ (when $x \in C_{p}$ ). Thus $w_{k}$ is the equivariant Stiefel Whitney class of the normal bundle to $C_{p}$ for $f_{\lambda a}$. Note that each of the functions in this perturbed family is still Morse-Bott-Kirwan, in the sense of Lemma 3.3, since in that lemma, we make no assumption on the value $a$.

Since $f_{\lambda a}(F)=\Phi(F) \cdot \Phi(F)+2 \lambda \Phi(F) \cdot \Phi\left(C_{p}\right)+\Phi\left(C_{p}\right) \cdot \Phi\left(C_{p}\right)$, we note that if $\Phi(F) \cdot \Phi\left(C_{p}\right)<0$, then for large enough $\lambda, f_{\lambda a}(F)<f_{\lambda a}\left(C_{p}\right)$. As $Q$ is compact, there is some $L$ such that $\lambda \geq L$ implies

$$
f_{\lambda a}(F)<f_{\lambda a}\left(C_{p}\right)
$$

for all connected components $F$ of $Q^{T_{\mathbb{R}}}$ with $\Phi(F) \cdot \Phi\left(C_{p}\right)<0$. Thus conditions (1)-(4) are satisfied by $h=f_{L a}$. Thus there exists $\beta \in K_{\mathbb{R}}$ and $\left.\beta_{p}\right|_{C_{i}}=\left.\alpha\right|_{C_{i}}$ for all $i \leq p$, as desired.

\section{EXAMPLES}

6.1. The product of two spheres. Let $\omega$ be the standard symplectic form on $\mathbb{C} P^{1}$, and let $S^{1}$ act on $\mathbb{C} P^{1}$ by rotation. Let $M=\mathbb{C} P^{1} \times \mathbb{C} P^{1}$ with symplectic form $(\omega,-\omega)$. Consider the Hamiltonian $T=S^{1}$ action on $M$ which sends

$$
\theta \cdot(z, w) \mapsto(\theta \cdot z,-\theta \cdot w) .
$$

Then the involution $\sigma$ which switches the factors of $M, \sigma(z, w)=(w, z)$ is antisymplectic and anti-commutes with the $S^{1}$ action. Moreover, the real locus of this involution is $Q=\{(w, w)\}$, the diagonal copy of $\mathbb{C} P^{1}$. The $T_{\mathbb{R}}$ action on $Q$ is an action by $\mathbb{Z} / 2 \mathbb{Z}$, where the nontrivial element acts by rotation by $\pi$. 
Let $\Phi$ be a moment map for the $S^{1}$ action on $M$, and let $\mu$ be a regular value. Then $M / / S^{1}(\mu)=\mathbb{C} P^{1}$ and $Q / / T_{\mathbb{R}}(\mu)=\mathbb{R} P^{1}$. Theorem 1 implies that there is a surjection

$$
\kappa_{\mathbb{R}}: H_{\mathbb{Z} / 2 \mathbb{Z}}^{*}\left(\mathbb{C} P^{1} ; \mathbb{Z} / 2 \mathbb{Z}\right) \rightarrow H^{*}\left(\mathbb{R} P^{1} ; \mathbb{Z} / 2 \mathbb{Z}\right)
$$

The real locus $\mathbb{C} P^{1}$ is equivariantly formal, since $H^{*}\left(\mathbb{C} P^{1} ; \mathbb{Z} / 2 \mathbb{Z}\right)$ has classes only in even degrees. Thus, as a vector space,

$$
H_{T_{\mathbb{R}}}^{*}\left(\mathbb{C} P^{1} ; \mathbb{Z} / 2 \mathbb{Z}\right) \cong H_{T_{\mathbb{R}}}^{*} \otimes H^{*}\left(\mathbb{C} P^{1} ; \mathbb{Z} / 2 \mathbb{Z}\right) .
$$

The only class in degree 1 is the equivariant class in $H_{T_{\mathbb{R}}}^{1}(p t ; \mathbb{Z} / 2 \mathbb{Z})$, and so the only element of $H_{T_{\mathbb{R}}}^{*}\left(\mathbb{C} P^{1} ; \mathbb{Z} / 2 \mathbb{Z}\right)$ which is not in the kernel of $\kappa_{\mathbb{R}}$ is this equivariant class.

6.2. A mod 2 GKM example. Suppose $M$ is a compact Hamiltonian $T$ space. Suppose further that $M$ has only finitely many fixed points, and that for $p \in M^{T}$, the weights of the isotropy representation of $T$ on $T_{p} M$ are pairwise independent over $\mathbb{Z}$ and over $\mathbb{Z} / 2 \mathbb{Z}$. Then we say that $M$ is a $\bmod 2 G K M$ space. Such examples include toric varieties, all coadjoint orbits in type $A_{n}$, and some (nonmaximal) coadjoint orbits in other types. In this case, a result of [6] states that there is an isomorphism

$$
H_{T}^{2 *}(M ; \mathbb{Z} / 2 \mathbb{Z}) \cong H_{T_{\mathbb{R}}}^{*}(Q ; \mathbb{Z} / 2 \mathbb{Z})
$$

dividing degrees in half. Moreover, if we write down each of these rings as subrings of the equivariant $\mathbb{Z} / 2 \mathbb{Z}$-cohomology of the appropriate fixed point sets, then the subrings are equal with a change in grading inherited from the module structure. Both the left- and right-hand sides of equation (6.1) are modules over the corresponding cohomology of a point. In both cases, this coefficient ring is the polynomial ring $\mathbb{Z} / 2 \mathbb{Z}\left[x_{1}, \ldots, x_{n}\right]$ but the generators are regarded as degree 2 classes on the left, and as degree 1 classes on the right. For example, suppose $M=\mathcal{O}_{\lambda}$ is a coadjoint orbit of type $A_{2}$. In Figure 1, we show an equivariant class $\alpha$, represented by a polynomial associated to each fixed point, pictured here on the moment map image. As a class in $H_{T}^{*}\left(\mathcal{O}_{\lambda} ; \mathbb{Z} / 2 \mathbb{Z}\right), \alpha$ is a degree 2 class. As a class in $H_{T_{\mathbb{R}}}^{*}\left(\left(\mathcal{O}_{\lambda}\right)^{\sigma} ; \mathbb{Z} / 2 \mathbb{Z}\right)$, it is a degree 1 class.

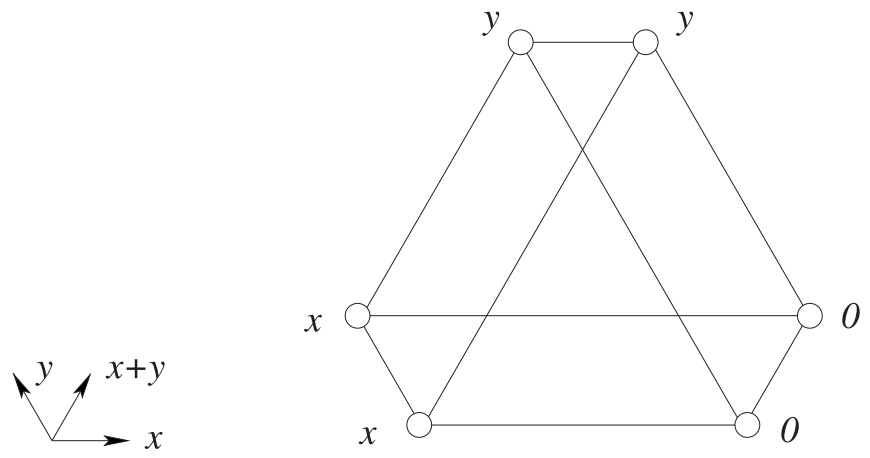

Figure 1. This shows an equivariant class $\alpha$ on a coadjoint orbit $\mathcal{O}_{\lambda}$ of type $A_{2}$.

Now let $\Phi: M \rightarrow \mathfrak{t}^{*}$ be the moment map, and suppose $\mu \in \mathfrak{t}^{*}$ is a regular value such that $T$ acts freely on $\Phi^{-1}(\mu)$. As a result of Kirwan's surjectivity for symplectic reductions and Theorem [1, we have two short exact sequences in equivariant 
cohomology, with $\mathbb{Z} / 2 \mathbb{Z}$ coefficients:

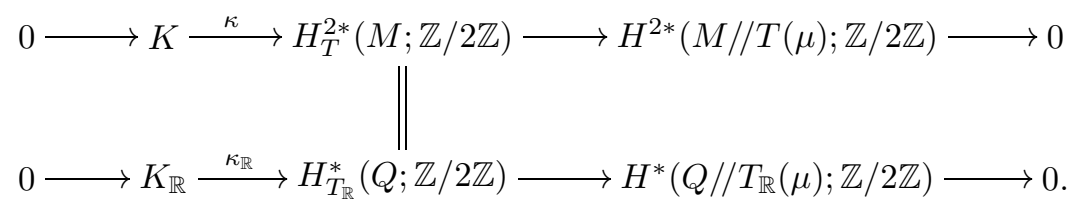

The Tolman-Weitsman Theorem and Theorem 2 imply that the generators of $K$ and $K_{\mathbb{R}}$ are identified under the equality. For example, Figure 2 demonstrates the class $\alpha$ which is a degree 2 class in $K_{\xi}(M)$ and is a degree 1 class in $K_{\xi}(Q)$, for the $\mu$ and $\xi$ shown.
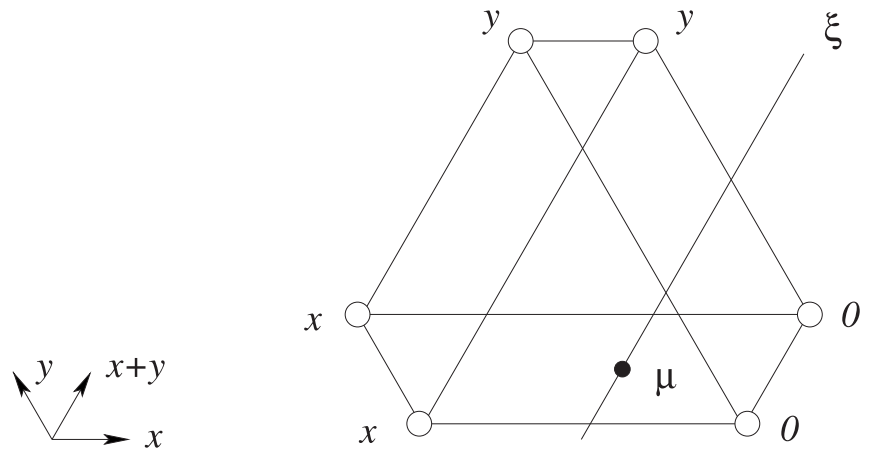

Figure 2. This shows a class $\alpha$ in $K_{\xi}$ for $\mathcal{O}_{\lambda} / / T(\mu)$ and for $\left(\mathcal{O}_{\lambda} / / T(\mu)\right)^{\sigma}$.

Thus, we have a commutative diagram

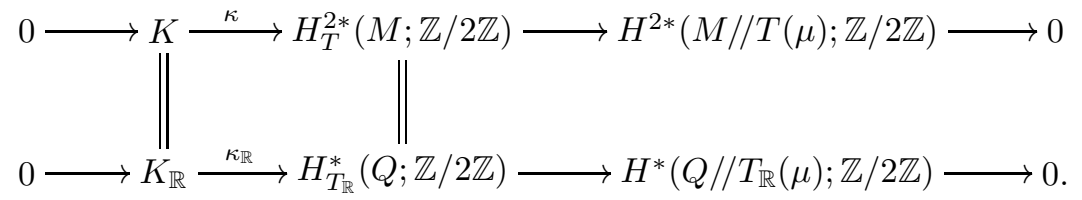

By the Five Lemma,

$$
H^{2 *}(M / / T(\mu) ; \mathbb{Z} / 2 \mathbb{Z})=H^{*}\left(Q / / T_{\mathbb{R}}(\mu) ; \mathbb{Z} / 2 \mathbb{Z}\right)
$$

as rings. This result is analogous to Duistermaat's original result on the topology of real loci; however, $M / / T$ may not have a Hamiltonian torus action. Thus this example extends Duistermaat's result to symplectic reductions.

6.3. Real toric varieties. In the symplectic setting, a toric variety is a compact symplectic $2 n$-dimensional manifold with an effective Hamiltonian $T^{n}$-action. Let the moment map be $\Phi: M \rightarrow \mathfrak{t}^{*}$. Then $\Phi(M)=\Delta$ is a simple rational convex polytope. Delzant [10] proved that there is a bijection between simple rational convex polytopes in $\mathbb{R}^{n}$ and toric varieties. For every simple rational convex polytope, Delzant constructed a toric variety $M_{\Delta}$, which is a symplectic reduction of affine space. Namely, $M_{\Delta}=\mathbb{C}^{d} / / T^{k}$.

Danilov [8] computed the ordinary cohomology of a toric variety in terms of combinatorial data encoded in its moment polytope. He showed that

$$
H^{*}\left(M_{\Delta} ; \mathbb{C}\right)=\mathbb{C}\left[x_{1}, \ldots, x_{k}\right] / I,
$$


where $I$ is an ideal of classes depending on how faces of $\Delta$ intersect. In this case, it is natural to think of the map

$$
H_{T^{k}}^{*}\left(\mathbb{C}^{d} ; \mathbb{C}\right)=\mathbb{C}\left[x_{1}, \ldots, x_{k}\right] \rightarrow H^{*}\left(M_{\Delta} ; \mathbb{C}\right)
$$

as the Kirwan map, and that it is a surjection follows by an argument similar to Kirwan's. While $\mathbb{C}^{d}$ is not compact, the compactness assumption can be replaced by the assumption that the moment map is proper and bounded in some direction. The action of $T^{k}$ on $\mathbb{C}^{d}$ certainly satisfies these assumptions. Indeed, an equivariant extension of Kirwan's surjectivity, due to the first author [12], can also be applied, giving a map

$$
H_{T^{d}}^{*}\left(\mathbb{C}^{d} ; \mathbb{C}\right) \rightarrow H_{T^{n}}^{*}\left(\mathbb{C}^{d} / / T^{k} ; \mathbb{C}\right),
$$

where $n=d-k$. The kernel computation does not go through in the same way, but we have an alternative description of $H_{T^{n}}^{*}\left(M_{\Delta} ; \mathbb{C}\right)$ which allows us to compute the kernel for real loci of toric varieties.

We can compute the equivariant cohomology of toric varieties using a combinatorial description due to Goresky, Kottwitz and MacPherson [13. Provided $M_{\Delta}$ has no 2-torsion, this combinatorial description also holds for real loci 6], and so we know that as rings,

$$
H_{T^{n}}^{2 *}\left(M_{\Delta} ; \mathbb{Z} / 2 \mathbb{Z}\right)=H_{T_{\mathbb{R}}^{n}}^{*}\left(M_{\Delta}^{\sigma} ; \mathbb{Z} / 2 \mathbb{Z}\right) .
$$

Moreover, this equality commutes with the two surjections, giving a commutative diagram

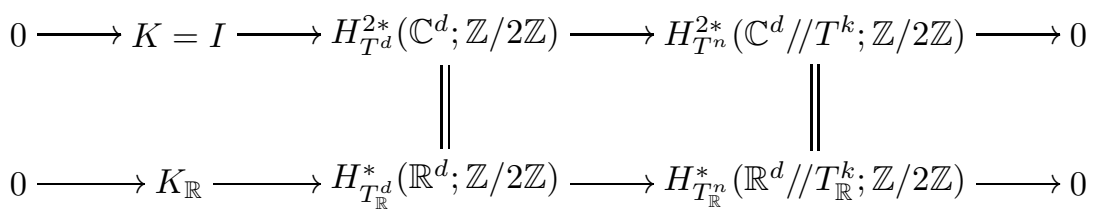

Thus, by the Five Lemma, $K=K_{\mathbb{R}}$. This description of the cohomology of $\left(M_{\Delta}\right)^{\sigma}$ as a quotient of a polynomial ring is identical to Danilov's description of the cohomology of $M_{\Delta}$. It was proved using other methods by Davis and Januszkievicz 9].

\section{REFERENCES}

[1] C. Allday and V. Puppe, Cohomological Methods in Transformation Groups, Cambridge Studies in Advanced Mathematics 32 1994, Cambridge University Press, Cambridge. MR 94g:55009

[2] M. Atiyah, Convexity and commuting Hamiltonians. Bull. London Math. Soc. 14 (1982), no. $1,1-15$.

[3] M. Atiyah and R. Bott, The moment map and equivariant cohomology. Topology 23 (1984), 1-28. MR 85e:58041

[4] M. Atiyah and R. Bott, Yang-Mills Equations over Riemann Surfaces. Philos. Trans. Roy. Soc. London A 308 (1982), 523-615. MR 85k:14006

[5] N. Berline and M. Vergne, Classes caractéristiques équivariantes. Formules de localisation en cohomologie équivariante. C.R. Acad. Sci. Paris Sér. I Math. 295 (1982), 539-541. MR 83m:58002

[6] D. Biss, V. Guillemin, and T. Holm, The mod 2 equivariant cohomology of fixed point sets of anti-symplectic involutions, to appear in Advances in Math. math.SG/0107151.

[7] T. Chang and T. Skjelbred, The topological Schur lemma and related results, Ann. of Math. (2) 100 (1974), 307-321. MR 51:11552

[8] V. Danilov, The geometry of toric varieties. Russian Math. Surveys 33 (1978), no. 2, 97-154. MR 80g:14001 
[9] M. Davis and T. Januszkiewicz. Convex polytopes, Coxeter orbifolds and torus actions, Duke Math. J. 62 (1991), no. 2, 417-451. MR 92i:52012

[10] T. Delzant, Hamiltoniens périodiques et images convexes de l'application moment. [Periodic Hamiltonians and convex images of the momentum mapping] Bull. Soc. Math. France 116 (1988), no. 3, 315-339. MR 90b:58069

[11] H. Duistermaat, Convexity and tightness for restrictions of Hamiltonian functions to fixed point sets of an anti-symplectic involution. Trans. Amer. Math. Soc. 275 (1983), no. 1, 417-429. MR 84c:53035

[12] R. F. Goldin, An effective algorithm for the cohomology ring of symplectic reductions, Geom. and Func. Anal. 12 (2002), 567-583.

[13] M. Goresky, R. Kottwitz, and R. MacPherson, Equivariant cohomology, Koszul duality, and the localization theorem, Invent. Math. 131 (1998), 25-83. MR 99c:55009]

[14] F. Kirwan, Cohomology of Quotients in Symplectic and Algebraic Geometry, Princeton University Press, Princeton, NJ, 1984. MR 86i:58050

[15] J. Milnor and J. Stasheff, Characteristic Classes, Princeton University Press, Princeton, NJ, 1974. MR 55:13428

[16] L. O'Shea and R. Sjamaar, Moment maps and Riemannian symmetric pairs. Math. Ann. 317 (2000), no. 3, 415-457. MR 2001g:53146

[17] C. Schmid, Cohomologie équivariante de certaines variétés hamiltoniennes et de leur partie réelle. Thèse at Université de Genève. Available at http://www.unige.ch/ biblio/these/theses.html

[18] E. Spanier, Algebraic Topology, McGraw-Hill, New York, 1966. MR 35:1007

[19] S. Tolman and J. Weitsman, The cohomology ring of symplectic quotients. Comm. Anal. Geom. 11 (2003), no. 4, 751-773.

[20] S. Tolman and J. Weitsman, On the cohomology rings of Hamiltonian T-spaces, Northern CA Symplectic Geometry Seminar, 251-258, Amer. Math. Soc. Transl. Ser. 2, 196, Amer. Math. Soc., Providence, RI, 1999. MR 2000j:57071

Mathematical Sciences, George Mason University, MS 3F2, 4400 University Dr., FairFAX, VIRGina 22030

E-mail address: rgoldin@math.gmu.edu

Department of Mathematics, University of California Berkeley, 813 Evans Hall, Berkeley, California 94720

E-mail address: tsh@math.berkeley.edu 